\title{
Embedded Metal Oxide Plasmonics Using Local Plasma Oxidation of AZO for Planar Metasurfaces
}

\author{
Kai Sun,* Wei Xiao, Sheng Ye, Nikolaos Kalfagiannis, Kian Shen Kiang, \\ C. H. (Kees) de Groot, * and Otto L. Muskens*
}

New methods for achieving high-quality conducting oxide metasurfaces are of great importance for a range of emerging applications from infrared thermal control coatings to epsilon-near-zero nonlinear optics. This work demonstrates the viability of plasma patterning as a technique to selectively and locally modulate the carrier density in planar Al-doped ZnO (AZO) metasurfaces without any associated topographical surface profile. This technique stands in strong contrast to conventional physical patterning which results in nonplanar textured surfaces. The approach can open up a new route to form novel photonic devices with planar metasurfaces, for example, antireflective coatings and multi-layer devices. To demonstrate the performance of the carrier-modulated AZO metasurfaces, two types of devices are realized using the demonstrated plasma patterning. A metasurface optical solar reflector is shown to produce infrared emissivity equivalent to a conventional etched design. Second, a multiband metasurface is achieved by integrating a Au visible-range metasurface on top of the planar AZO infrared metasurface. Independent control of spectral bands without significant cross-talk between infrared and visible functionalities is achieved. Local carrier tuning of conducting oxide films offers a conceptually new approach for oxide-based photonics and nanoelectronics and opens up new routes for integrated planar metasurfaces in optical technology.

Metal oxides are technologically highly important materials with many applications including oxide electronics, ${ }^{[1-4]}$ solar cells, ${ }^{[5]}$ chemical sensors, ${ }^{[6-9]}$ and catalysis. ${ }^{[8]}$ Transparent conducting oxides (TCOs) such as indium tin oxide (ITO) and Al-doped $\mathrm{ZnO}$ (AZO) are large bandgap doped semiconductors with a typical density of free electrons in the range of $10^{19}-10^{21} \mathrm{~cm}^{-3}$ in between that of doped semiconductors and noble metals. The optical response of TCOs can be described accurately using the Drude model for free electrons and is characterized by a dielectric behavior in the visible range with a transition to metallic behavior in the infrared (IR) spectral range. ${ }^{[10,11]}$ The density of free electrons can be controlled by adding electron donors ${ }^{[12-14]}$ and depends on material processing conditions..$^{[5,10,15]}$

In recent years, the field of TCOs as advanced materials for photonics applications has emerged to exploit their unique optical response in between the regimes of metals and dielectrics and their tunability through design and through elec-

Dr. K. Sun, W. Xiao, Prof. O. L. Muskens

Astronomy and Physics

Faculty of Engineering and Physical Sciences

University of Southampton

Southampton SO17 1BJ, UK

E-mail:k.sun@soton.ac.uk; o.muskens@soton.ac.uk

Dr. K. Sun, W. Xiao, Dr. S. Ye, Dr. K. S. Kiang, Prof. C. H. (K.) de Groot

Electronics and Computer Science

Faculty of Engineering and Physical Sciences

University of Southampton

Southampton SO17 1BJ, UK

E-mail: chdg@ecs.soton.ac.uk

Dr. N. Kalfagiannis

Department of Physics and Mathematics

School of Science and Technology

Nottingham Trent University

Nottingham NG11 8NS, UK

The ORCID identification number(s) for the author(s) of this article can be found under https://doi.org/10.1002/adma.202001534.

(C) 2020 The Authors. Published by WILEY-VCH Verlag GmbH \& Co. KGaA, Weinheim. This is an open access article under the terms of the Creative Commons Attribution License, which permits use, distribution and reproduction in any medium, provided the original work is properly cited.

DOI: 10.1002/adma.202001534 trical or all-optical control. ${ }^{[15-22]}$ Of particular interest is their use in metasurfaces, thin two-dimensional metamaterial layers that are used to navigate electromagnetic waves. ${ }^{[21,23-26]}$ The fundamental challenge in processing TCO's is concurrently obtaining thin films with favorable electrical and optical conditions. For instance, to design efficient, transparent conductors for photovoltaic and conductive display applications, relatively low resistivity, and optical transparency have to be achieved. For electro-optic and nonlinear applications, the epsilon-near-zero (ENZ) response around the bulk plasmon frequency has been under intense investigation. ${ }^{[27-29]}$ Moreover, ITO films could enable an ENZ circuit board and nanoscale structured ITO resistor/capacitor/inductor (RCL) equivalent circuit elements, thus creating a viable means to realize metatronic circuits. ${ }^{[30]}$

AZO is a highly desirable material for its low cost and environmental friendly nontoxicity ${ }^{[31]}$ and therefore is rapidly becoming the most used TCO in practical applications. AZO can be formed through a wide range of deposition techniques. ${ }^{[32-36]}$ Among these techniques, the atomic layer deposition (ALD) process has specific advantages related to its lower process temperature, excellent uniformity, accurate thickness control as well as high reproducibility owing to the surface-saturated and self-limiting reaction mechanism. The Al 
content in $\mathrm{ZnO}$ can be uniformly and accurately controlled through the ratio control of $\mathrm{Al}_{2} \mathrm{O}_{3}$ cycle and $\mathrm{ZnO}$ cycle.

In order to establish AZO as a versatile material for photonics, tunability of the bulk plasmon frequency through the modulation of carrier concentration is desirable. Tuning of the carrier concentrations in the AZO film can be achieved for example through the $\mathrm{Al}$ content, the film thickness, deposition temperature, ${ }^{[37,38]}$ and laser annealing. ${ }^{[39]}$ Tuning of the $\mathrm{Al}$ content through the ratio of $\mathrm{Al}_{2} \mathrm{O}_{3}$ and $\mathrm{ZnO}$ cycles has been intensively studied and it was found that the carrier concentration increases with the $\mathrm{Al}$ content in the range of $0-5 \% .37,40]$ Other methods of carrier tuning have been shown for pure $\mathrm{ZnO}$ produced by ALD without Al dopants, where an in situ $\mathrm{O}_{2}$ plasma treatment reduced the carrier concentration, ${ }^{[41,42]}$ while an in situ $\mathrm{H}_{2}$ plasma treatment increased the carrier concentration. ${ }^{[43]}$ Furthermore, no works have been reported to our knowledge on plasma treatment for modulating the carrier density in $\mathrm{AZO}$, which bears more interest than pure $\mathrm{ZnO}$ for its applications in IR optical metasurfaces.

In this work, we demonstrate that local patterning of carrier density in $\mathrm{AZO}$ films through an $\mathrm{O}_{2}$ plasma treatment enables the formation of planar IR metasurfaces that lack any associated surface topography. The approach stands in strong contrast to physical patterning such as plasma etching, ${ }^{[44,45]}$ controlled evaporation, ${ }^{[46]}$ and lift-off, ${ }^{[47,48]}$ all of which result in non-planar textured surfaces which significantly influences the planarity of subsequent device layers. Therefore, the proposed technique can open up a new route to form novel photonic devices through its selective AZO optical property modulation. We demonstrate the performance of $\mathrm{O}_{2}$ plasma patterned AZO using two examples of device applications. The first demonstrator is an optical solar reflector (OSR) device, while the second application introduces a multi-band metasurface obtained by depositing a visible Au antenna array on top of the planar AZO metasurface, showing independent operation in the visible and IR ranges. Overall we find good performance of the devices, demonstrating the feasibility of the approach as a versatile new tool for obtaining high quality patterned metasurfaces without requiring a physical structuring of the materials.

AZO film of $100 \mathrm{~nm}$ thickness was deposited by thermal ALD at a temperature of $175{ }^{\circ} \mathrm{C}$ using diethylzinc (DEZ), trimethylaluminum (TMA), and $\mathrm{H}_{2} \mathrm{O}$ precursors. The $\mathrm{Al}$ doping of $\mathrm{ZnO}$ is controlled by the $\mathrm{Al} / \mathrm{Zn}$ precursor cycle ratio during the film growth. ${ }^{[24,49]}$ Following the film deposition, the carrier density can be ex situ modulated by exposing the film to an $\mathrm{O}_{2}$ plasma for up to $20 \mathrm{~min}$ at a temperature of $300{ }^{\circ} \mathrm{C}$. The influence of temperature was investigated and very little influence of the carrier density was found for AZO films treated at lower temperatures, as shown in Section S1, Supporting Information. The effect of plasma treatment time is shown in Section S2, Supporting Information and reveals a gradual reduction in carrier density over treatment times from 0-20 min. Hall Effect measurements were performed on the plasma treated AZO films, resulting in values for the carrier density as shown in Figure 1a against the $\mathrm{Al} / \mathrm{Zn}$ doping ratio. The plasma treatment is effective in significantly reducing the carrier concentration for all $\mathrm{Al} /$ $\mathrm{Zn}$ ratios under study. For $\mathrm{Al} / \mathrm{Zn}$ ratios of $0-4 \%$, the carrier concentration was reduced by nearly five orders of magnitude from $10^{21}$ to $10^{16} \mathrm{~cm}^{-3}$. For the highest $\mathrm{Al} / \mathrm{Zn}$ ratio of $5 \%$, the reduction in order of magnitude is less strong, but as this doping ratio corresponds to the highest overall carrier density, the absolute reduction in carrier concentration is actually the largest.
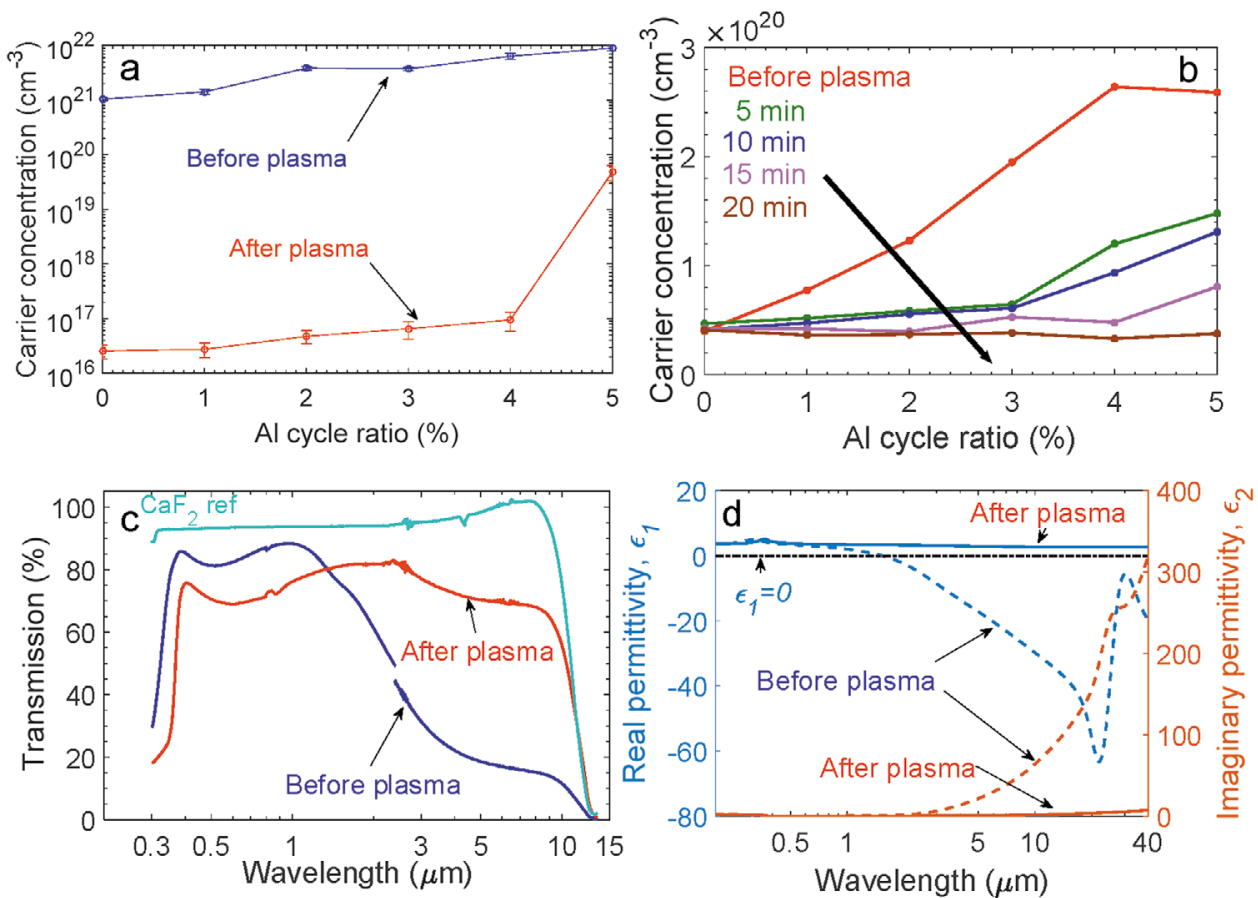

Figure 1. Plasma-treated Al-doped $\mathrm{ZnO}$ (AZO) film characterizations. a) Carrier concentration of AZO films after exposure to $\mathrm{O}_{2}$ plasma for 20 min as determined by the Hall effect with the lines being guides to the eye, b) carrier concentration of AZO films for various $\mathrm{O}_{2}$ plasma exposure times as extracted from ellipsometry with the lines being guides to the eye, c) transmission spectra of $4 \% \mathrm{AZO}$ on $\mathrm{CaF}_{2}$ before and after a 20 min $\mathrm{O}_{2}$ plasma treatment, and d) permittivity ( $\varepsilon_{1}$ and $\varepsilon_{2}$ ) of $4 \% \mathrm{AZO}$ on $\mathrm{CaF}_{2}$ before and after a $20 \mathrm{~min} \mathrm{O}_{2}$ plasma treatment extracted from ellipsometry. 
Figure $1 \mathrm{~b}$ shows the carrier density extracted from variable angle spectroscopic ellipsometry measurements, where Drude model ${ }^{[50]}$ fits over the range of $600-1700 \mathrm{~nm}$. In the case of pure $\mathrm{ZnO}$, the extracted carrier concentration is $\leq 5 \times 10^{19} \mathrm{~cm}^{-3}$ as the optical measurements are insensitive to carrier concentrations below this concentration. Therefore the optical response of the pure $\mathrm{ZnO}$ appears unchanged by the $\mathrm{O}_{2}$ plasma treatment of up to $20 \mathrm{~min}$. For the $\mathrm{AZO}$ films with an $\mathrm{Al} / \mathrm{Zn}$ ratio between $1 \%$ and $5 \%$, carrier concentrations reach up to $2.7 \times 10^{20} \mathrm{~cm}^{-3}$ prior to the $\mathrm{O}_{2}$ plasma treatment. The subsequent exposure to $\mathrm{O}_{2}$ plasma results in a reduction of the carrier density to below the sensitivity limit of $5 \times 10^{19} \mathrm{~cm}^{-3}$ and the $20 \mathrm{~min}$ plasma treatment time is therefore considered to be the optimized duration for optical device applications, beyond which optical property contrast is unlikely to be further improved. Corresponding model fits are shown in Section S3, Supporting Information. A marked difference in measured carrier concentration of nearly one order of magnitude is seen between the ellipsometry and Hall Effect measurements in Figure 1. A similar discrepancy is also seen in other work ${ }^{[38]}$ and is generally attributed to a difference in carrier densities participating in DC electrical conductivity and high-frequency optical conductivity in these materials. In this work, we demonstrate optical applications of the plasma treatment technique and therefore we refer in the remainder of this work to the optical carrier density which is extracted from the ellipsometry. A full investigation of the mechanism that the $\mathrm{O}_{2}$ plasma exposure reduces the carrier concentration of $\mathrm{ZnO}$ and $\mathrm{Al}$-doped $\mathrm{ZnO}$ is beyond the scope of this work though some insights are discussed in Section S4, Supporting Information.

The AZO carrier densities up to $2.7 \times 10^{20} \mathrm{~cm}^{-3}$ are well matched to a range of applications in mid-infrared thermal control metasurfaces covering the long-wave IR at $>8 \mu \mathrm{m}$ wavelength. To further increase the carrier density of the AZO for mid-wave IR $(<10 \mu \mathrm{m})$ applications, we modified the ALD process to a higher substrate temperature of $250{ }^{\circ} \mathrm{C}$, resulting in a significantly increased optical carrier density of up to $6 \times 10^{20} \mathrm{~cm}^{-3}$. Figure 1c shows the transmission of a resulting AZO film at a doping ratio of $4 \%$, deposited onto a calcium fluoride $\left(\mathrm{CaF}_{2}\right)$ substrate of $1 \mathrm{~mm}$ thickness, before and after a $20 \mathrm{~min}$ exposure to $\mathrm{O}_{2}$ plasma. The $\mathrm{CaF}_{2}$ substrate has a transmission cut-off around $10 \mu \mathrm{m}$. Again we clearly see the effect of the plasma exposure on the IR transmission, which is characterized by the metallic Drude response before treatment. A high visible-range transmission of about $70 \%$ is maintained following the plasma treatment, which extends all the way to the near infrared (NIR) and IR ranges indicating again a significant carrier density reduction of the AZO.

The exact optical constants, in terms of real and imaginary permittivity, $\varepsilon_{1}$ and $\varepsilon_{2}$, were extracted through appropriate modelling employing two independent spectroscopic ellipsometry measurements in the visible (Vis) and IR $(1.2-40 \mu \mathrm{m})$ spectral ranges for the corresponding AZO film with $4 \%$ doping ratio deposited at $250{ }^{\circ} \mathrm{C}$, before and after the $20 \mathrm{~min} \mathrm{O}_{2}$ plasma treatment. The optical constants are shown in Figure 1d. In the wavelength range between 200 and $1500 \mathrm{~nm}, \varepsilon_{1}$ and $\varepsilon_{2}$ remain mostly unaffected by the plasma treatment. In the IR range above $1500 \mathrm{~nm}, \varepsilon_{1}$ and $\varepsilon_{2}$ are significantly modulated. Before the plasma treatment, $\varepsilon_{1}$ crosses over zero in the IR range, indicating the AZO is metallic at IR range whilst is dielectric at visible range. After plasma treatment, $\varepsilon_{1}$ is above zero at all measurement ranges, indicating a switch in the metallic character of AZO to a pure dielectric behavior from 0.2 to $40 \mu \mathrm{m}$. This is consistent with $\varepsilon_{2}$ trend which is significantly reduced at IR range after the plasma treatment. The above optical constants were used in numerical simulations of demonstrator devices presented further below. In all cases, the whole depths of AZO films are considered to be impacted by the $20 \mathrm{~min} \mathrm{O}_{2}$ plasma treatment since no thin high carrier AZO film can be identified from optical measurements.

To demonstrate the performance of the AZO metasurfaces obtained using $\mathrm{O}_{2}$ plasma, we first evaluated a demonstrator device aimed at radiative cooling of spacecraft. OSRs play a crucial role in the thermal control of a spacecraft. ${ }^{[24,51,52]}$ OSRs are designed to reflect the solar radiation and radiatively dissipate the heat that is generated on board. Optically, an OSR is a spectrally selective filter that reflects the radiation spectrum of the sun and emits the thermal infrared spectrum corresponding to that of a blackbody at $300 \mathrm{~K}$. We show here that our planar metasurface AZO optical solar reflector (meta-OSR) can be formed with an equivalent optical functionality to a conventional non-planar metasurface. ${ }^{[24,51]}$

The successful modulation of carrier concentration through an $\mathrm{O}_{2}$ plasma shown above now allows us to develop a plasma patterning technique to selectively and locally modulate AZO carrier density. To achieve patterning, we use a silicon nitride (SiN) hard mask as high temperature oxygen diffusion barrier ${ }^{[53]}$ (see Experimental Section and Section S5, Supporting Information for details). Figure 2a shows the schematic of the meta-OSR based on the plasma patterning technique. The AZO is deposited at $175{ }^{\circ} \mathrm{C}$ on top of a $\mathrm{SiO}_{2} / \mathrm{Al}$ stack to create a $\lambda / 4$ perfect absorber stack. Patterning of the SiN hard mask by electron beam lithography and plasma etching is carried out to prepare the mask for selective plasma oxidation. Application of the $\mathrm{O}_{2}$ plasma exposure to the AZO sample covered by the patterned hard mask results in a strong reduction in the AZO carrier density in the exposed region (marked as L or low), leaving the carrier concentration in the unexposed AZO region intact (marked as $\mathrm{H}$ or high). The carrier concentration contrast is thus defined by the hard mask pattern. After plasma treatment, the SiN layer is removed with little damage to the layer underneath and an optically patterned AZO metasurface is engineered that is fully embedded inside a physically planar surface. In comparison, the metasurface OSR formed by a conventional lithography and plasma etch of AZO is schematically shown in Figure $2 \mathrm{~b}$ and is similar to results presented previously by us. ${ }^{[24]}$

Figure 2c,d shows the scanning electron microcopy (SEM) images for the $\mathrm{O}_{2}$ plasma patterning and conventional etch, respectively. Through the greyscale contrast, the square shape array of dimensions of about $700 \times 700 \mathrm{~nm}^{2}$ can be identified, more clearly in the etched films than the plasma treated films. Atomic force microscopy (AFM) 2D mapping (Figure 2e,f) and corresponding cutline AFM profiles (Figure 2g,h) clearly demonstrate the difference between the two approaches. The conventionally etched pattern shows as expected clear topographic difference between the AZO pattern and the $\mathrm{SiO}_{2}$ with $80 \mathrm{~nm}$ height difference as extracted from the AFM cutline. The $\mathrm{O}_{2}$ plasma exposure does not affect the height profile and a continuous planar film remains intact, although with some increased 

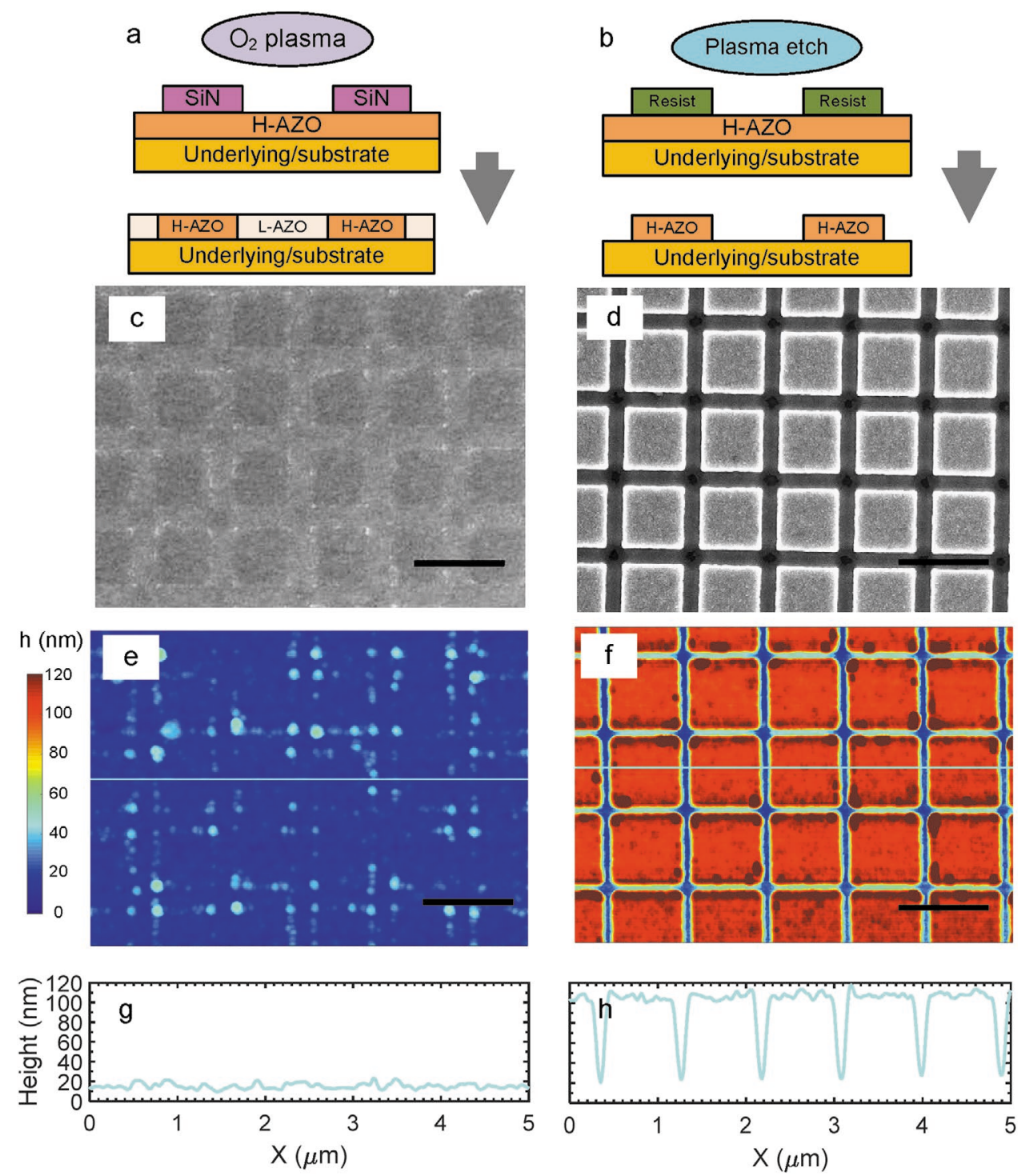

Figure 2. Metasurface comparison formed by plasma patterning and conventional etch techniques. a,b) Schematic of the meta-OSR and resulting AZO features after plasma patterning and conventional etch, c,d) SEM image of meta-OSR of $700 \mathrm{~nm}$ features and $250 \mathrm{~nm}$ gap by plasma patterning and conventional etch, e,f) AFM map of the meta-OSR by plasma patterning and conventional etch, and g,h) cutline profile in AFM image of (e,f). The scale is $1 \mu \mathrm{m}$ for both SEM and AFM figures.

roughness and minor impurities with topography below $10 \mathrm{~nm}$ being observed. Those impurities, shown as white dots in the SEM, and light blue in the AFM, are attributed to polymer residue.

The optical response of the meta-OSRs formed by plasma patterning and conventional etch, respectively, are shown in Figure 3a,b. For both meta-OSRs, the FTIR absorption spectrum shows a significantly broader absorption at around 7 and $12 \mu \mathrm{m}$ than that of the OSR stack without metasurface patterning (labeled as film). This effect is attributed to the broadband plasmonic enhancement in this spectral range as detailed in the previous work and resulting in an increased blackbody emissivity. ${ }^{[24]}$ The slight discrepancy in spectra between plasma patterning and etch meta-OSRs can be attributed to the optical difference between low carrier AZO and air. The difference in absorption spectra between the patterned and unpatterned AZO films translates into a significant increase of the IR emissivity $(\varepsilon)$ of these meta-OSRs from 0.65 for the unpatterned AZO structure to 0.81 for both $\mathrm{O}_{2}$ plasma and etch patterned plasmonic structures. The IR emissivity is a radiation cooling performance indicator of an OSR and further detail on IR emissivity definition and calculation is available in our previous work. ${ }^{[24]}$ The excellent agreement between plasma-patterned and etch patterned reflector spectra unambiguously shows that plasma-patterning technique can be used as an alternative route to form metasurface whilst maintaining a physically planar film. This is highly advantageous for further device processing such as the addition of an antireflective coating stack.

To further demonstrate the versatility of the plasma patterning technique and extend our work to mid-wave IR 


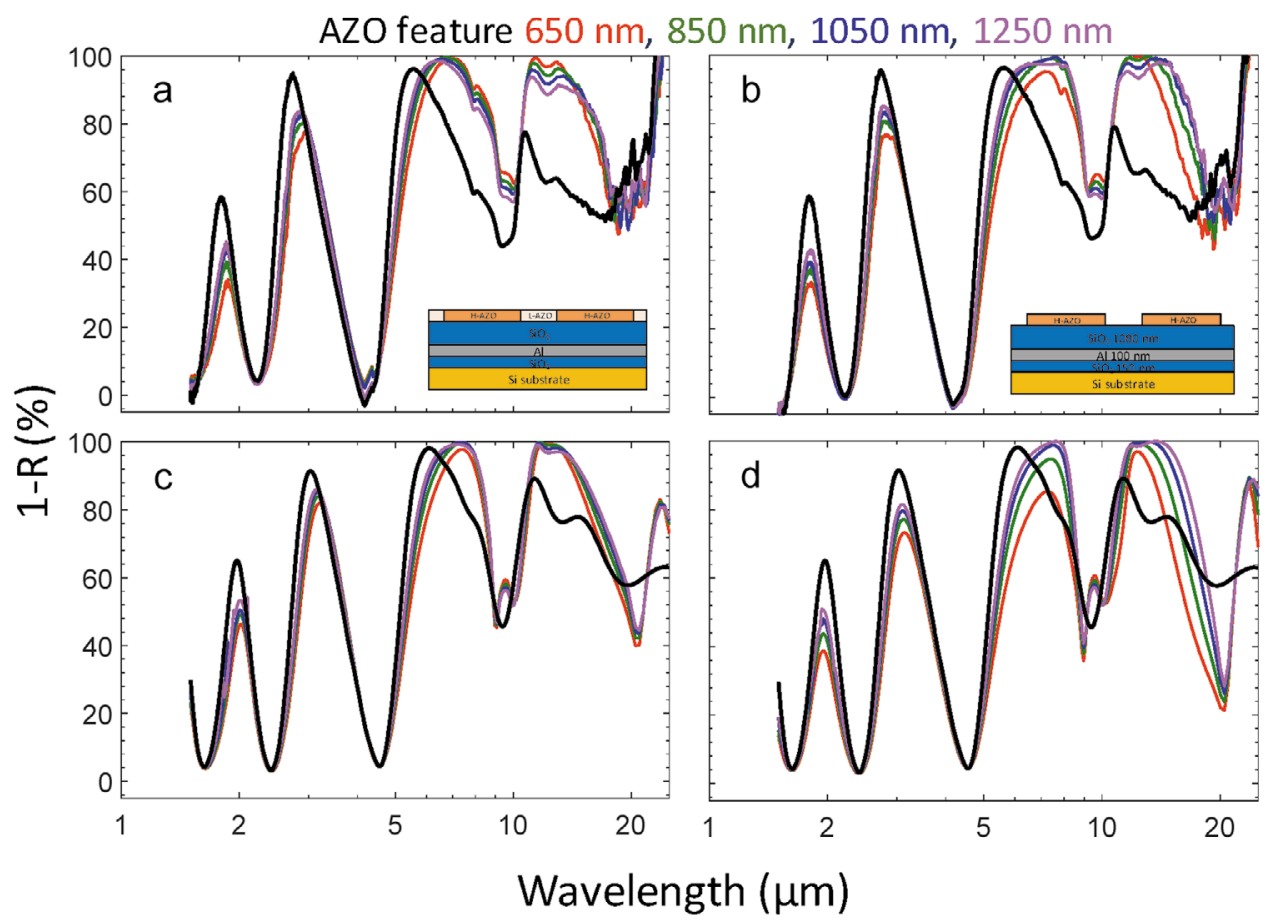

Figure 3. Optical characterization and comparison between plasma patterned and conventional etched metasurfaces. a,b) FTIR absorption spectra of meta-OSRs for varying AZO feature size and constant $250 \mathrm{~nm}$ gap by plasma pattern and etch pattern. c,d) Simulated absorption spectra of (a) and (b). Solid black line in all figures is the identical spectra for the planar AZO film without any patterning.

applications, we developed a second demonstrator device showing the capability of achieving multiband metasurfaces with independent functionalities in IR and visible parts of the spectrum. The multiband metasurface device, or multi-meta device in short, with multiplexing spectral functionalities is realized by integrating an embedded planar AZO metasurface for infrared applications with an $\mathrm{Au}$ metasurface for visiblerange applications. The fabrication of the proposed multi-meta devices is schematically shown in Figure $4 \mathrm{a}$ and is further detailed in Section S6, Supporting Information. The AZO metasurface consists of a $100 \mathrm{~nm} \mathrm{AZO} \mathrm{(Al/Zn} \mathrm{ratio} \mathrm{4 \% )} \mathrm{atop} \mathrm{a} \mathrm{CaF}_{2}$ substrate deposited at $250{ }^{\circ} \mathrm{C}$ to achieve a high carrier density. The film is again patterned using the established method of $\mathrm{O}_{2}$ plasma exposure through a lithographically patterned $\mathrm{SiN}$ hard mask. After the SiN removal, an array of $40 \mathrm{~nm}$ thick gold antennas is formed through evaporation and lift-off on top of the embedded AZO metasurfaces. AZO and Au lithography layers are non-commensurate and no intentional alignment is applied.

Figure $4 \mathrm{~b}$ shows an SEM image of the fabricated multi-meta device with a high magnification image shown as inset. The metasurface combines an Au feature size of $120 \mathrm{~nm} \times 320 \mathrm{~nm}$ and a square AZO feature of $1340 \mathrm{~nm}$ in size. SEM images of other metasurfaces with different dimensions of the $\mathrm{Au}$ and AZO components are presented in Section S7, Supporting Information. The key feature of the SEM image is that the Au antenna geometry is unaffected by the AZO pattern underneath, independent of where it is located relatively to the AZO pattern. In particular, some antennas are positioned over the edge of an AZO feature, but their shapes remain intact. This is confirmed by the AFM image of the same multiband metasurface shown in Figure 4c, with Figure 4d showing two cutline profiles marked in the AFM color map. The AZO feature can be barely identified in the AFM map, indicating a planar surface, whilst $\mathrm{Au}$ antennas can be easily seen in bright color. The cutline 1 is through the Au antennas and AZO features and the profile shows predominantly the Au antenna. A periodic step of about $40 \mathrm{~nm}$ in height is seen consistent with the Au antenna thickness. No systematic shape or topography change is found for $\mathrm{Au}$ antennas inside, outside, or partially overlapping with the AZO features. By comparison, in the cutline 2 over the AZO feature without $\mathrm{Au}$ antenna, a tiny indentation $(<10 \mathrm{~nm})$ can be identified at the position of the gaps. Therefore, the AZO metasurface formed by the plasma patterning technique is confirmed to be planar and exerting little or no impact on the subsequent deposited $\mathrm{Au}$ antennas. Therefore, the planar metasurface is highly advantageous as there is no restraint of pitch match or alignment between two functional arrays.

Optical transmission spectra for our multiband metasurfaces are presented in the Vis and IR range for the fabricated device with different combinations of feature sizes. Vis spectra were taken using polarized light along the longitudinal E-field with respect to antenna direction. The independent infrared tunability is verified by varying the AZO feature size from 340 to $1840 \mathrm{~nm}$ while keeping the Au antenna length fixed at $240 \mathrm{~nm}$ by $120 \mathrm{~nm}$. Experimental transmission spectra are shown in Figure $5 \mathrm{a}$ for the Vis range and Figure $5 \mathrm{~b}$ for IR range (unpolarized), with corresponding simulations presented in Figure $5 c, d$. The $\mathrm{CaF}_{2}$ substrate spectra is plotted as reference. The Vis functionality of the devices shows as the predominant the halfwavelength mode of the Au antenna of fixed dimension, and the AZO features only slightly affect the spectra with a small 

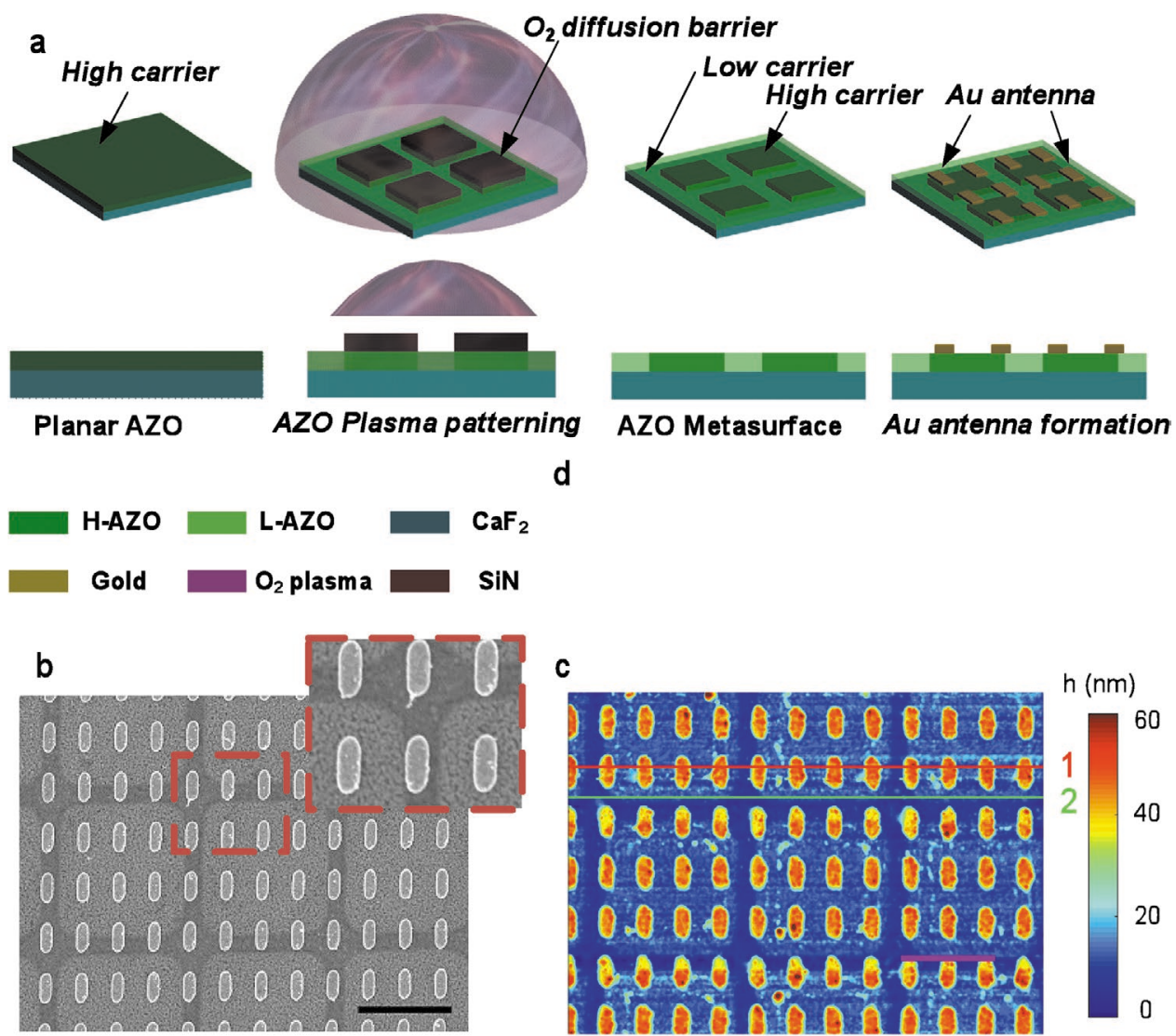

Figure 4. Multi-meta device formation and characterizations. a) Schematic of multi-meta device fabrication, b) SEM image of the fabricated multimeta device with Au plasmonic features on an AZO metasurface and Au pattern of $320 \mathrm{~nm}$ feature with $220 \mathrm{~nm}$ gap vertical and $120 \mathrm{~nm}$ feature with $220 \mathrm{~nm}$ gap horizontal. AZO square features of 1340 and $160 \mathrm{~nm}$ gap, c) AFM image of the multi-meta device, and d) cutline profiles through Au and AZO features (Cutline 1, red) and AZO feature only (Cutline 2, green). Scale bar in (b) and (c) is $1 \mu \mathrm{m}$.

decrease in the resonance width seen in both experiment and simulation. We note that the simulations required the design to be commensurate within the unit cell of the AZO metasurface in order to apply periodic boundary conditions. Tunability of the IR spectrum is seen with AZO feature size (Figure $5 b, d)$, where the cutoff at $>10 \mu \mathrm{m}$ corresponds to the $\mathrm{CaF}_{2}$ substrate. Overall experiments show a $20 \%$ higher IR transmission for experiments than simulations. This could be attributed to potential overestimations of the coupling with longitudinal plasmon resonance in the numerical simulation, which was taken for plane wave incidence.

Independent tunability of the visible-range band is demonstrated by fixing the AZO feature size at $1340 \mathrm{~nm}$ while varying the $\mathrm{Au}$ antenna length from 150 to $320 \mathrm{~nm}$ (with a fixed width of $120 \mathrm{~nm}$ ). Figure 5e (visible range) and Figure $5 \mathrm{f}$ (IR range) show the experimental results, and Figure $5 \mathrm{~g}, \mathrm{~h}$ the corresponding numerical simulations. The antenna resonance shifts from 0.8 to $1.1 \mu \mathrm{m}$ with $\mathrm{Au}$ feature size increasing from 150 to $320 \mathrm{~nm}$, while the transverse mode does not shift with antenna length (not shown). The different arrays show near identical spectra in the IR spectra, indicating that the IR response is unaffected by the Au metasurface. Therefore, multiband metasurface devices based on the plasma patterning technique are demonstrated to have independent optical tunability at Vis and IR bands, owning to lack of both topographic and optical interaction between the planar AZO IR metasurface and Au visible-range metasurface.

In summary, we reported that optical properties of Al-doped $\mathrm{ZnO}$ films can be strongly modulated by a free carrier modulation reduction through exposure to an $\mathrm{O}_{2}$ plasma. A modulation up to 5 orders of magnitude is demonstrated through electrical and optical characterizations. Based on these results, we demonstrate a plasma-patterning technique to selectively and locally modulate AZO carrier density without resulting in a topographical profile. A planar metasurface AZO meta-optical solar reflector (meta-OSR) can be formed with an equivalent optical functionality to a conventional non-planar metasurface. We also reported the successful fabrication of a novel multimeta device consisting of two separate AZO and $\mathrm{Au}$ metasurfaces, which independently operate in visible and IR ranges.

The proposed plasma patterning technique opens up a new route to form novel photonic devices by enabling a selective optical property modulation of AZO. Other applications could be found in transparent electrodes, invisible metasurfaces for product labelling and anti-counterfeiting. Vertical depth profile of the carrier concentration modulation of the films is uniform in our devices but could potentially be controlled by grey scale lithography to create a whole new category of materials. ${ }^{[54]}$ Other materials amenable to local carrier concentration include $\mathrm{TiO}_{2}{ }^{[55]}$ or 2D transition metal dichalcogenides (TDMC) such as 


\section{Au feature $\mathrm{H} 240 \mathrm{~nm} / \mathrm{W} 120 \mathrm{~nm}$ and AZO feature $340 \mathrm{~nm}, 840 \mathrm{~nm}, 1340 \mathrm{~nm}$ and $1840 \mathrm{~nm}$}
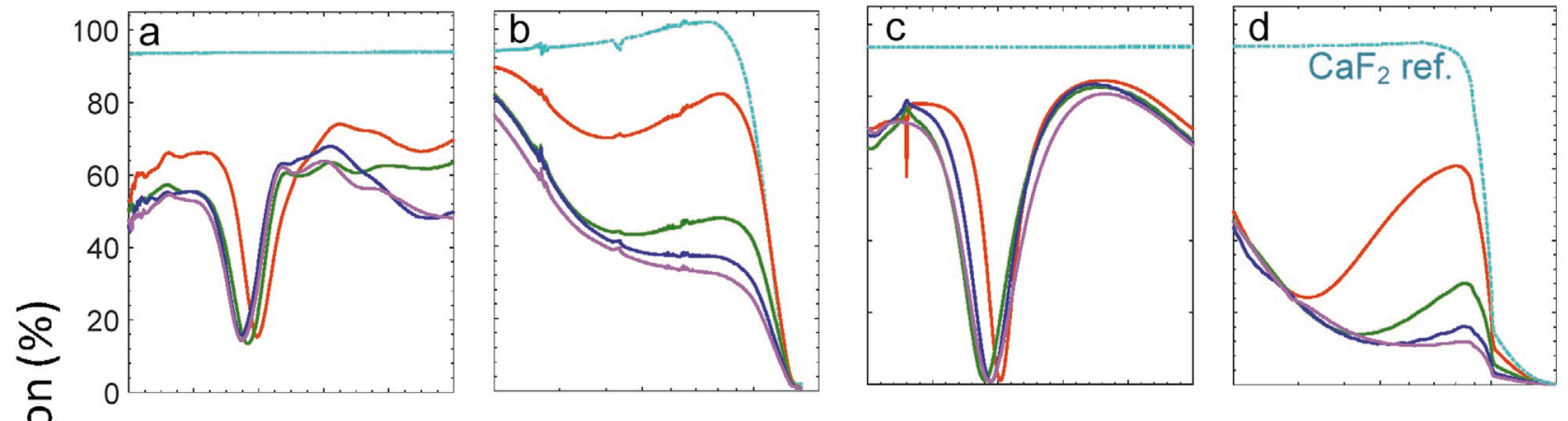

\section{AZO feature $1340 \mathrm{~nm}$ and Au feature height: $150 \mathrm{~nm}, 190 \mathrm{~nm}, 240 \mathrm{~nm}$ and $320 \mathrm{~nm}$}
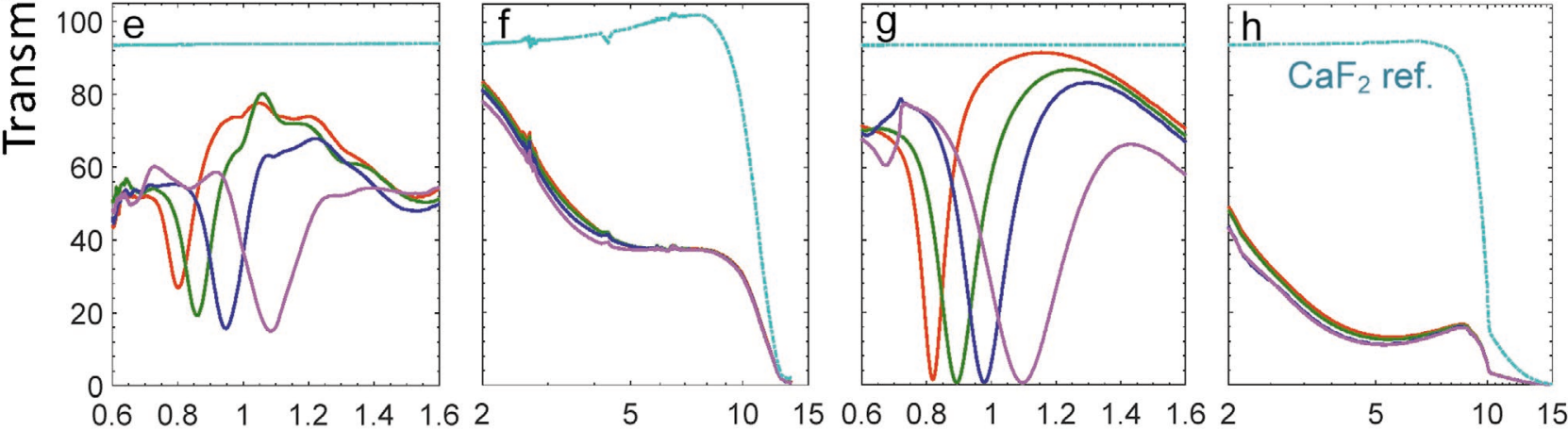

Wavelength $(\mu \mathrm{m})$

Figure 5. Multi-meta devices with independent functionalities at different wavelength ranges through Au feature and AZO feature variations. a-d) Transmission spectra of the multi-metasurface devices with a fixed Au feature $(L \times W$ as $240 \mathrm{~nm} \times 120 \mathrm{~nm})$ and various AZO square features with width ranging from 340 to $1840 \mathrm{~nm}$ for: a) experimental Vis range, b) experimental IR range, c) simulated Vis range, and d) simulated IR range. e-h) Transmission spectra of the multi-band metasurface devices with a fizzed AZO feature of $1340 \mathrm{~nm}$ and Au features ranging from 150 to $320 \mathrm{~nm}$ vertical by $120 \mathrm{~nm}$ horizontal for: e) experimental Vis range, f) experimental IR range, g) simulated Vis range, and h) simulated IR range. CaF ${ }_{2}$ reference is given for all graphs.

$\mathrm{MoS}_{2}{ }^{[56,57]}$ which could be the topic of future investigations. We believe that plasma patterning techniques have broad potentials for electronic and photonic applications to achieve novel structures once unimaginable or beyond the existing manufacture capability.

\section{Experimental Section}

Plasma Exposure and Plasma Patterning Technique: A $100 \mathrm{~nm}$ AZO film was deposited using a Savannah S200 thermal ALD system with TMA, DEZ and $\mathrm{H}_{2} \mathrm{O}$ precursors. Al doping was controlled by adjusting the $\mathrm{Al} / \mathrm{Zn}$ cycle ratios from $0 \%$ to $5 \%$ (detailed in previous work ${ }^{[24]}$ ). The resulting $\mathrm{AZO}$ film was then exposed to an $\mathrm{O}_{2}$ plasma at an elevated temperature of $300{ }^{\circ} \mathrm{C}$ in an Oxford Instruments FlexAl ALD system, equipped with an inductively coupled plasma (ICP) source for high density plasma. The $\mathrm{O}_{2}$ plasma condition was set at an $\mathrm{O}_{2}$ flow of $60 \mathrm{sccm}$, Pressure of $150 \mathrm{mTorr}$ and ICP power of $400 \mathrm{~W}$. To eliminate the effect of annealing at $300{ }^{\circ} \mathrm{C}$ without plasma source, the total time in the chamber is $26 \mathrm{~min}$ for all samples independent of $\mathrm{O}_{2}$ plasma exposure time.

For the plasma patterning, the samples were capped with a $3 \mathrm{~nm}$ $\mathrm{Al}_{2} \mathrm{O}_{3}$ by ALD as a protection layer after AZO deposition step and then a $80 \mathrm{~nm}$ SiN hard mask layer was deposited by plasma-enhanced chemical vapor deposition (PECVD) at $200{ }^{\circ} \mathrm{C}$. The SiN layer was subsequently patterned by e-beam lithography using a JEOL JBX-9300FS e-beam system and then an ion beam etch process using an Oxford Instrument lonFab 300 Plus system. $\mathrm{A} \mathrm{CHF}_{3}+\mathrm{Ar}$ etch process was used for SiN layer patterning whilst the AZO film was patterned using a pure Ar etch process for etch pattern samples. The resist (ZEP520A) was then stripped using a low temperature $\mathrm{O}_{2}$ ICP plasma removal with sample's temperature well controlled to be below $65^{\circ} \mathrm{C}$ through short term cycled plasma etch and cooling and the sample was exposed to $\mathrm{O}_{2}$ plasma as previous described. After the plasma treatment, the SiN was selectively removed using a specially developed ICP process of fluorine chemistry with a low ion damage.

Optical Characterizations: Infrared reflectance was measured over the range of 2.5 to $15 \mu \mathrm{m}$ using a Fourier transform infrared microscope (Thermo-Nicolet Nexus 670, Continuum microscope) with a $15 \times$ optical objective with a numerical aperture of 0.58 and a MCT detector. The $\mathrm{KBr}$ beamsplitter and IR source was used and the transmittance and reflectance were normalized with air and aluminum mirror, respectively. A separate visible-range spectrophotometer was used to cover the spectrum from 0.6 to $2.0 \mu \mathrm{m}$ for the gold metasurface arrays. An $80 \mathrm{~nm}$ aluminum coated $\mathrm{SiO}_{2} / \mathrm{Si}$ substrate was used as reference for reflection. The Vis/NIR optical property of the AZO films was characterized using an ellipsometry system (J.A.Woollam M-2000) with a spectral range of 200 to $1700 \mathrm{~nm}$. The IR Ellipsometry measurements were performed using a Woollam IR variable angle spectroscopic Ellipsometer Mark II with a spectral range of 1.25 to $40 \mu \mathrm{m}$. The AZO film was modelled as 
film with an uniform carrier density over the whole depth. The AZO carrier density was extracted employing an appropriate Drude model..50]

Numerical Modeling: The simulations of the meta-OSRs and multimeta devices were done using the finite difference time domain (FDTD) method implemented in Lumerical software. Spectra from 0.3-30 $\mu \mathrm{m}$ wavelength were obtained using a broadband short pulse source. The source was a plane wave incident normal to the surface. Symmetric and anti-symmetric boundary conditions were used to reduce the computation volume. The refractive index and extinction coefficients of AZO were derived from Ellipsometry using the Drude model, other materials including $\mathrm{Au}, \mathrm{SiO}_{2}, \mathrm{Al}, \mathrm{SiN}$, and $\mathrm{CaF}_{2}$ are from tabulated references. ${ }^{[58]}$ Linearly polarized incident light was used for metaOSRs, and polarized light along the longitudinal E-field with respect to antenna direction was used for multiband devices in the visible spectrum. Unpolarized light was used where stated for the IR response of the multiband devices, and was obtained by averaging over the two orthogonal polarizations.

\section{Supporting Information}

Supporting Information is available from the Wiley Online Library or from the author.

\section{Acknowledgements}

K.S. and O.L.M. acknowledge the funding from DASA/Dstl under Grant Agreement No. DSTLX1000130136 "MULTIMETA". O.L.M. acknowledges the funding from EPSRC Program grant on Metadevices and Metasystems, EP/M009122/1. W.X. acknowledges the funding support from the China Scholarship Council. N.K. acknowledges support from Nottingham Trent University capital equipment funding scheme for acquiring the Infrared Spectroscopic Ellipsometry. All data supporting this study are openly available from the University of Southampton repository at DOI: https://doi.org/10.5258/SOTON/D1263.

\section{Conflict of Interest}

The authors, K.S., C.H.D.G. and O.L.M., have filed a patent on the plasma patterning technique with United Kingdom Patent Application No. 1913533.4, "Method to Create Lateral Modulation of Optical and Electronic Properties of Semiconducting Metal Oxides or Chalcogenides by Masked Plasma Exposure".

\section{Keywords}

aluminum zinc oxide, carrier modulation, metal oxide, multi metasurfaces, optical solar reflectors, oxygen plasma, plasma patterning, transparent conductive oxides

Received: March 3, 2020

Revised: April 19, 2020

Published online:

[1] Z. Zulkifli, M. Subramanian, T. Tsuchiya, M. S. Rosmi, P. Ghosh, G. Kalita, M. Tanemura, RSC Adv. 2014, 4, 64763.

[2] S. M. Chen, S. S. Wang, Appl. Phys. Lett. 2014, 105, 223304.

[3] R. Waser, M. Aono, Nat. Mater. 2007, 6, 833.

[4] E. N. Dattoli, Q. Wan, W. Guo, Y. Chen, X. Pan, W. Lu, Nano Lett. 2007, 7, 2463.

[5] C. G. Granqvist, Sol. Energy Mater. Sol. Cells 2007, 91, 1529.
[6] D. Caruntu, K. Yao, Z. X. Zhang, T. Austin, W. L. Zhou, C. J. O'Connor, J. Phys. Chem. C 2010, 114, 4875.

[7] K. Sun, I. Zeimpekis, C. Hu, N. M. J. Ditshego, O. Thomas, M. R. R. de Planque, H. M. H. Chong, H. Morgan, P. Ashburn, Microelectron. Eng. 2016, 153, 96.

[8] A. Kolmakov, M. Moskovits, Annu. Rev. Mater. Res. 2004, 34, 151.

[9] E. Comini, C. Baratto, G. Faglia, M. Ferroni, A. Vomiero, G. Sberveglieri, Prog. Mater. Sci. 2009, 54, 1.

[10] S. H. Brewer, S. Franzen, J. Phys. Chem. B 2002, 106, 12986.

[11] P. P. Edwards, A. Porch, M. O. Jones, D. V. Morgan, R. M. Perks, Dalton Trans. 2004, 2004, 2995.

[12] Z. H. Lu, J. X. Zhou, A. J. Wang, N. Wang, X. N. Yang, J. Mater. Chem. 2011, 21, 4161.

[13] E. Hammarberg, A. Prodi-Schwab, C. Feldmann, J. Colloid Interface Sci. 2009, 334, 29.

[14] A. R. Babar, P. R. Deshamukh, R. J. Deokate, D. Haranath, C. H. Bhosale, K. Y. Rajpure, J. Phys. D: Appl. Phys. 2008, 41, 135404.

[15] G. V. Naik, V. M. Shalaev, A. Boltasseva, Adv. Mater. 2013, 25, 3264.

[16] M. Abb, P. Albella, J. Aizpurua, O. L. Muskens, Nano Lett. 2011, 11, 2457.

[17] M. Abb, Y. Wang, N. Papasimakis, C. H. de Groot, O. L. Muskens, Nano Lett. 2014, 14, 346

[18] R. Amin, J. K. George, S. Sun, T. F. d. Lima, A. N. Tait, J. B. Khurgin, M. Miscuglio, B. J. Shastri, P. R. Prucnal, T. El-Ghazawi, V. J. Sorger, APL Mater. 2019, 7, 081112.

[19] M. Abb, Y. Wang, C. H. de Groot, O. L. Muskens, Nat. Commun. 2014, 5, 4869.

[20] P. Guo, R. D. Schaller, J. B. Ketterson, R. P. H. Chang, Nat. Photonics 2016, 10, 267.

[21] Y.-W. Huang, H. W. H. Lee, R. Sokhoyan, R. A. Pala, K. Thyagarajan, S. Han, D. P. Tsai, H. A. Atwater, Nano Lett. 2016, 16, 5319.

[22] Y. Wang, A. Capretti, L. Dal Negro, Opt. Mater. Express 2015, 5, 2415.

[23] H. Caglayan, S.-H. Hong, B. Edwards, C. R. Kagan, N. Engheta, Phys. Rev. Lett. 2013, 111, 073904.

[24] K. Sun, C. A. Riedel, Y. Wang, A. Urbani, M. Simeoni, S. Mengali, M. Zalkovskij, B. Bilenberg, C. H. de Groot, O. L. Muskens, ACS Photonics 2018, 5, 495.

[25] S. A. Gregory, Y. Wang, C. H. de Groot, O. L. Muskens, ACS Photonics 2015, 2, 606.

[26] G. Kafaie Shirmanesh, R. Sokhoyan, R. A. Pala, H. A. Atwater, Nano Lett. 2018, 18, 2957.

[27] N. Kinsey, C. DeVault, J. Kim, M. Ferrera, V. M. Shalaev, A. Boltasseva, Optica 2015, 2, 616.

[28] O. Reshef, I. De Leon, M. Z. Alam, R. W. Boyd, Nat. Rev. Mater. 2019, 4, 535.

[29] A. Capretti, Y. Wang, N. Engheta, L. Dal Negro, ACS Photonics 2015, 2, 1584.

[30] I. Liberal, N. Engheta, Nat. Photonics 2017, 11, 149.

[31] R. R. Biswal, A. Maldonado, M. D. Olvera, presented at the 11th Int. Conf. on Electrical Engineering, Computing Science and Automatic Control, Campeche, Mexico, October 2014.

[32] M. Hiramatsu, K. Imaeda, N. Horio, M. Nawata, J. Vac. Sci. Technol., A 1998, 16, 669.

[33] Y. Igasaki, H. Kanma, Appl. Surf. Sci. 2001, 169-170, 508.

[34] A. Martin, J. P. Espinos, A. Justo, J. P. Holgado, F. Yubero, A. R. Gonzalez-Elipe, Surf. Coat. Technol. 2002, 151-152, 289.

[35] M. Ohyama, H. Kozuka, T. Yoko, J. Am. Ceram. Soc. 1998, 81, 1622.

[36] S. M. Sultan, O. D. Clark, T. B. Masaud, Q. Fang, R. Gunn, M. M. A. Hakim, K. Sun, P. Ashburn, H. M. H. Chong, Microelectron. Eng. 2012, 97, 162.

[37] N. P. Dasgupta, S. Neubert, W. Lee, O. Trejo, J. R. Lee, F. B. Prinz, Chem. Mater. 2010, 22, 4769.

[38] A. K. Pradhan, R. M. Mundle, K. Santiago, J. R. Skuza, B. Xiao, K. D. Song, M. Bahoura, R. Cheaito, P. E. Hopkins, Sci. Rep. 2014, 4,6415 . 
[39] S. O. El hamali, W. M. Cranton, N. Kalfagiannis, X. Hou, R. Ranson, D. C. Koutsogeorgis, Opt. Lasers Eng. 2016, 80, 45.

[40] T. Tynell, H. Yamauchi, M. Karppinen, R. Okazaki, I. Terasaki, J. Vac. Sci. Technol., A 2013, 31, 1.

[41] M. A. Thomas, J. B. Cui, ACS Appl. Mater. Interfaces 2012, 4, 3122.

[42] R. Huang, S. Ye, K. Sun, K. S. Kiang, C. H. de Groot, Nanoscale Res. Lett. 2017, 12, 541.

[43] T. R. Sharp, A. K. Peter, B. MacKenzie, H. C. M. Knoops, R. Gunn, presented at the 14th Int. Conf. on Atomic Layer Deposition, Kyoto, Japan, June 2014.

[44] G. Q. Zhang, C. W. Lan, H. L. Bian, R. Gao, J. Zhou, Opt. Express 2017, 25, 22038.

[45] S. Colburn, A. Zhan, A. Majumdar, Sci. Adv. 2018, 4, eaar2114.

[46] M. Hentschel, M. Schaferling, X. Y. Duan, H. Giessen, N. Liu, Sci. Adv. 2017, 3, e1602735.

[47] J. Burch, D. D. Wen, X. Z. Chen, A. Di Falco, Sci. Rep. 2017, 7, 4520.

[48] Y. D. Wang, M. Abb, N. Papasimakis, C. H. de Groot, O. L. Muskens, presented at the 2014 Conf. on Lasers and ElectroOptics, San Jose, CA, USA, June 2014.

[49] A. K. Pradhan, R. M. Mundle, K. Santiago, J. R. Skuza, B. Xiao, K. D. Song, M. Bahoura, R. Cheaito, P. E. Hopkins, Sci. Rep. 2015, 4,6415 .
[50] Optical Properties of Solids, (Ed: F. Wooten), Academic Press, Burlington, MA, USA 1972, p. 42.

[51] K. Sun, C. A. Riedel, A. Urbani, M. Simeoni, S. Mengali, M. Zalkovskij, B. Bilenberg, C. H. de Groot, O. L. Muskens, ACS Photonics 2018, 5, 2280.

[52] D. U. Yildirim, A. Ghobadi, M. C. Soydan, O. Atesal, A. Toprak, M. D. Caliskan, E. Ozbay, ACS Photonics 2019, 6, 1812.

[53] V. D. Kunz, T. Uchino, C. H. de Groot, P. Ashburn, D. C. Donaghy, S. Hall, Y. Wang, P. L. F. Hemment, IEEE Trans. Electron Devices 2003, 50, 1487.

[54] Y. Chen, X. Duan, M. Matuschek, Y. Zhou, F. Neubrech, H. Duan, N. Liu, Nano Lett. 2017, 17, 5555.

[55] Y. Wu, W. Yang, Y. Fan, Q. Song, S. Xiao, Sci. Adv. 2019, 5, eaax0939.

[56] J. Jadwiszczak, C. O'Callaghan, Y. Zhou, D. S. Fox, E. Weitz, D. Keane, C. P. Cullen, I. O'Reilly, C. Downing, A. Shmeliov, P. Maguire, J. J. Gough, C. McGuinness, M. S. Ferreira, A. L. Bradley, J. J. Boland, G. S. Duesberg, V. Nicolosi, H. Zhang, Sci. Adv. 2018, 4, eaao5031.

[57] P. M. Campbell, C. J. Perini, J. Chiu, A. Gupta, H. S. Ray, H. Chen, K. Wenzel, E. Snyder, B. K. Wagner, J. Ready, E. M. Vogel, 2D Mater. 2017, 5, 015005.

[58] Handbook of Optical Constants of Solids, (Ed: E. D. Palik), Academic Press, Burlington, MA, USA 1997, p. xvii. 